\title{
Algorithmic correspondence for intuitionistic modal mu-calculus, Part 1 (Extended Abstract)
}

\author{
Willem Conradie $^{1}$, Yves Fomatati ${ }^{2}$, Alessandra Palmigiano ${ }^{2}$ \\ , and Sumit Sourabh ${ }^{2}$ \\ 1 University of Johannesburg, South Africa \\ wconradie@uj.ac.za \\ 2 ILLC, University of Amsterdam, The Netherlands \\ A.Palmigiano@uva.nl, S.Sourabh@uva.nl
}

\section{Algebraic-algorithmic correspondence}

Modal mu-calculus [5] is a logical framework combining simple modalities with fixed point operators, enriching the expressivity of modal logic so as to deal with infinite processes like recursion. It has a simple syntax, an easily given semantics, and is decidable. Many expressive modal and temporal logics such as PDL, CTL and CTL* can be seen as fragments of the modal mu-calculus.

Sahlqvist-style frame-correspondence theory for modal mu-calculus has recently been developed in [6]. The correspondence results in [6] are developed purely model-theoretically. However, they can be naturally encompassed within the existing algebraic approach to correspondence theory [2, 3, 4], and generalized to mu-calculi on a weaker-than-classical (and, particularly, intuitionistic) base. We focus in particular on the language of bi-intuitionistic modal mu-calculus, and we enhance the algorithm, or calculus for correspondence, ALBA [3] for the elimination of monadic second order variables, so as to guarantee its success over a class including the Sahlqvist mu-formulas defined in [6].

The algorithm ALBA: an example. Consider the transitivity axiom $\square p \rightarrow \square \square p$. As is discussed at length in [3, 2], every piece of argument used to prove this correspondence on frames can be translated by duality to their complex algebras, which are perfect distributive lattices with operators. The validity condition on frames translates into its complex algebra as $\mathbb{A} \vDash \forall p[\square p \leq \square \square p]$, followed by the chain of equivalences

$$
\begin{array}{ll} 
& \mathbb{A} \vDash \forall p[\square p \leq \square \square p] \\
\text { iff } & \mathbb{A} \vDash \forall p\left[\bigvee\left\{i \in J^{\infty}(\mathbb{A}) \mid i \leq \square p\right\} \leq \bigwedge\left\{m \in M^{\infty}(\mathbb{A}) \mid \square \square p \leq m\right\}\right] \\
\text { iff } & \mathbb{A} \vDash \forall p \forall \mathbf{i} \forall \mathbf{m}[(\mathbf{i} \leq \square p \& \quad \square p \leq \mathbf{m}) \Rightarrow \mathbf{i} \leq \mathbf{m}] \\
\text { iff } & \mathbb{A} \vDash \forall p \forall \mathbf{i} \forall \mathbf{m}[(\bullet \mathbf{i} \leq p \quad \& \quad \square p \leq \mathbf{m}) \Rightarrow \mathbf{i} \leq \mathbf{m}] \\
\text { iff } & \mathbb{A} \vDash \forall \mathbf{i} \forall \mathbf{m}[(\square \square \bullet \mathbf{i} \leq \mathbf{m}) \Rightarrow \mathbf{i} \leq \mathbf{m}]
\end{array}
$$

In above quasi-equations, $J^{\infty}(\mathbb{A})\left(\right.$ resp. $M^{\infty}(\mathbb{A})$ ) is the set of completely join-prime (resp. completely meet-prime) elements of the lattice, $\mathbf{i}$ ranging over $J^{\infty}(\mathbb{A})\left(\right.$ resp. $\mathbf{m}$ ranging over $M^{\infty}(\mathbb{A})$ ) is a nominal (resp. conominal) and $\bullet$ is the left adjoint of the $\square$ operation. The above equivalences hold because of the order-theoretic properties of the algebraic interpretation of the logical connectives. The last step, where the variable $p$ is eliminated, is a consequence of the Ackermann's lemma (cf. [3, lemma 4.2]). The final condition can be translated in the first order frame correspondence language (cf. [3, Section $6.3])$. 


\section{Extending algebraic-algorithmic approach to mu-calculus}

The syntactic manipulations presented in the example above can be described in terms of "proof rules" or rules of a rewriting system of a calculus for correspondence that is discussed in [3]. These rules come in two types: approximation and adjunction rules. Their soundness is based on the order theoretic properties of the interpretation of the logical connectives in the language. In order to extend this approach to mu calculus, we need to define sound adjunction and approximation rules for mu-formulas. The soundness is taken care of by the following lemma

Lemma 2.1. For $L$ and $M$ complete lattices and $G: M \times L \rightarrow L$, let $\mu y . G: M \rightarrow L$ be the map given by $a \mapsto L F P(G(a, y))$ for each $a \in M$ such that $L F P(G(a, y))$ is defined,

1. If $G$ is completely join-preserving, then $\mu y . G: M \rightarrow L$ is defined everywhere on $M$, and is completely join-preserving.

2. If, moreover, $F=\left(F_{1}, F_{2}\right): L \rightarrow M \times L$ is the right adjoint of $G$, then the right adjoint of $\mu y . G$ is the map defined by the assignment $z \mapsto v y .\left(F_{1}(y) \wedge F_{1}(z)\right)$.

\subsection{Approximation and general adjunction rules for fixed-point formulas}

Let AtProp and FVar be disjoint sets of propositional variables and of fixed point variables, and Var = AtProp $\cup$ FVar. Let $\mathcal{L}^{+}$be the expanded modal mu-language built over Var, nominals and conominals. Let $\epsilon$ be an order-type on an $n$-tuple $\bar{x}$ and $\overline{\mathbf{i}}_{i}{ }^{\epsilon}$ be the $n$-tuple whose $i$-th coordinate is $\mathbf{i}^{\epsilon_{i}}$ and whose $j$-th coordinate is $\perp^{\epsilon_{j}}$ for all $j \neq i \square^{1}$ We have the following approximation rule for the least fixed point operator

$$
\frac{\mathbf{i} \leq \mu X . \psi(\bar{\varphi} / \bar{x}, X, \bar{z})}{\mho_{i=1}^{n}\left(\exists \mathbf{j}_{i}{ }_{i}\left[\mathbf{i} \leq \mu X . \psi\left(\overline{\mathbf{j}}_{i}^{\epsilon} / \bar{x}, X, \bar{z}\right) \& \mathbf{j}^{\epsilon_{i}} \leq^{\epsilon_{i}} \varphi_{i}\right]\right)}\left(\mu^{\epsilon}-\mathrm{A}\right)
$$

where the tuples $\bar{x}$ and $\bar{z}$ are disjoint, and the variables $\bar{x} \in \operatorname{Var}$ do not occur in any formula in $\bar{\psi}$ or in $\bar{\varphi}$ and the associated term function of $\psi(\bar{x}, X, \bar{z})$ is completely $\bigvee$-preserving in $(\bar{x}, X) \in \mathbb{C}^{\epsilon} \times \mathbb{C}$, for any perfect modal bi-Heyting algebra $\mathbb{C}$. The following adjunction rule follows as an immediate consequence of lemma 2.12 .

$$
\frac{\mu X . \psi(\bar{\psi} / \bar{x}, X, \bar{z}) \leq \chi}{\&_{i=1}^{n} \psi_{i} \leq{ }^{\epsilon_{i}} v X .\left(G_{i}(\chi / y, \bar{z}) \wedge G_{i}(X / y, \bar{z})\right)}\left(\mu^{\epsilon} \text {-Adj }\right)
$$

where $\psi, \bar{\psi}, \chi \in \mathcal{L}^{+}$, the arrays of variables $\bar{x}$ and $\bar{z}$ are disjoint, $\bar{x}$ has arity $n$, the term function associated with $\psi(\bar{x}, X, \bar{z})$ is a left adjoint in $(\bar{x}, X) \in \mathbb{C}^{\epsilon} \times \mathbb{C}$ for any perfect modal bi-Heyting algebra $\mathbb{C}$, and $G=\left(\left(G_{i}(y, \bar{z})\right)_{i=1}^{n}, G^{\prime}(y, \bar{z})\right): \mathbb{C} \rightarrow \mathbb{C}^{\epsilon} \times \mathbb{C}$ is its right adjoint.

The order-theoretic lemma and rules for the greatest fixed point operator are given dually.

\subsection{Inner formulas and adjunction rules}

The adjunction rules as given above are too general, because they do not provide any information on know how to express $G_{i}$ in terms of the (extended) mu-language. The following definition is tailored to ensure that for any perfect modal bi-Heyting algebra $L$, the term function associated with a $\mathrm{IF}_{\delta}^{\square}$ (respectively, $\mathrm{IF}_{\delta}^{\diamond}$ ) formula is a right (respectively, left) adjoint from $L^{\delta} \rightarrow L$ fixing the variables $\bar{z}$ as parameters (see lemma below).

\footnotetext{
${ }^{1}$ Of course, if we fix a value for $\mathbf{i}^{\epsilon_{i}}$, then $\overline{\mathbf{i}}_{i}^{\epsilon}$ denotes the element in $J^{\infty}\left(\mathbb{A}^{\epsilon}\right)$ corresponding to $\mathbf{i}^{\epsilon_{i}}$ in the $i$-th coordinate.
} 
Definition 2.2. Let $\bar{y}, \bar{z} \subseteq$ Var and $\bar{X} \subseteq F$ Var be tuples of variables which are pairwise different in the union of the their underlying sets. Let $\delta$ be an order-type on $\bar{x}=\bar{y} \oplus \bar{X}$. The $\delta$ - $\square$ and $\delta-\diamond(\bar{x}, \bar{z})$-inner formulas $\left((\bar{x}, \bar{z})-I F_{\delta}^{\square}\right.$ and $\left.(\bar{x}, \bar{z})-I F_{\delta}^{\diamond}\right)$, the free variables of which are contained in $(\bar{x}, \bar{z})$, are given by the following simultaneous recursion (for the sake of readability, the parameters $\bar{x}$ and $\bar{z}$ are omitted):

$$
\begin{array}{lllllllll|l|l}
\mathrm{IF}_{\delta}^{\square} \ni \varphi & ::=x_{i} & \square \varphi & \varphi_{1} \wedge \varphi_{2} & v Y . \varphi^{\prime} & \pi \rightarrow \varphi & \pi \vee \varphi & \psi^{c} \rightarrow \pi \\
\mathrm{IF}_{\delta}^{\ominus} \psi & ::=x_{i} & \diamond \psi & \psi_{1} \vee \psi_{2} & \mu Y . \psi^{\prime} & \psi-\pi & \pi \wedge \psi & \pi-\varphi^{c}
\end{array}
$$

where $\delta_{i}=1$ in the base of the recursion, $\pi$ is a positive formula, $\varphi^{\prime}=\varphi^{\prime}\left(\bar{y} \oplus \bar{X}^{\prime}, \bar{z}\right)$ and $\psi^{\prime}=\psi^{\prime}\left(\bar{y} \oplus \bar{X}^{\prime}, \bar{z}\right)$ are $\mathrm{IF}_{\delta^{\prime}}^{\square}$ and $\mathrm{IF}_{\delta^{\prime}}^{\diamond}$, respectively, with $\bar{X}^{\prime}=\bar{X} \oplus Y$ and $\delta^{\prime}=\delta \oplus 1, \psi^{c} \in(\bar{x}, \bar{z})-\mathrm{IF}_{\delta^{\partial}}^{\diamond}$ and $\varphi^{c} \in(\bar{x}, \bar{z})-\mathrm{IF}_{\delta^{\partial}}^{\square}$. All other formulas have their free variables among $(\bar{x}, \bar{z})$.

The above definition also guarantees that the approximation rules $\left(\mu^{\delta}-\mathrm{A}\right)$ and $\left(v^{\delta}-\mathrm{A}\right)$ can be respectively applied in particular to inequalities of the form $\mathbf{i} \leq \mu X . \psi(\bar{y}, X, \bar{z})$ and $v X . \varphi(\bar{y}, X, \bar{z}) \leq \mathbf{m}$, such that $\mu X . \psi$ and $v X . \varphi$ are $(\bar{y}, \bar{z})-\mathrm{IF}_{\delta}^{\diamond}$ and $(\bar{y}, \bar{z})-\mathrm{IF}_{\delta}^{\square}$ sentences respectively.

The following definition of normal formulas is given in order to achieve an effective computation of adjoints of $\mathrm{IF}_{\delta}^{\square}$ and $\mathrm{IF}_{\delta}^{\diamond}$ formulas.

Definition 2.3. The normal $(\bar{x}, \bar{z})-\mathrm{IF}_{\delta}^{\square}$ and $(\bar{x}, \bar{z})-\mathrm{IF}_{\delta}^{\diamond}$ formulas are given by the same simultaneous recursion as in definition 2.2, subject to the following additional constraints:

1. if $\varphi$ is of the form $v Y \cdot \varphi^{\prime}\left(\bar{x}^{\prime}, \bar{z}\right)$, where $\bar{x}^{\prime}=\bar{y} \oplus \bar{X}^{\prime}$ and $\bar{X}^{\prime}=\bar{X} \oplus Y$, then there exists an $\left(\bar{y}^{\prime} \oplus \bar{X}^{\prime}, \bar{z}\right)-\mathrm{IF}_{\delta^{\prime}}$ formula $\varphi^{\prime \prime}$, where $\delta^{\prime}$ is the order-type over $\bar{y}^{\prime} \oplus \bar{X}^{\prime}$ which is constantly 1 over $\bar{y}^{\prime}$ and restricts to $\delta$ over $\bar{X}^{\prime}$, such that $\varphi^{\prime}\left(\bar{x}^{\prime}, \bar{z}\right)=\varphi^{\prime \prime}\left(\bar{\varphi} / \bar{y}^{\prime}, \bar{X}^{\prime}, \bar{z}\right)$ where the $\bar{\varphi}$ are normal $(\bar{y}, \bar{z})-\mathrm{IF}_{\delta^{\prime \prime}}^{\square}$ sentences, where $\delta^{\prime \prime}$ is the restriction of $\delta$ to $\bar{y}$.

2. if $\psi$ is of the form $\mu Y \cdot \psi^{\prime}\left(\bar{x}^{\prime}, \bar{z}\right)$, where $\bar{x}^{\prime}=\bar{y} \oplus \bar{X}^{\prime}$ and $\bar{X}^{\prime}=\bar{X} \oplus Y$, then there exists an $\left(\bar{y}^{\prime} \oplus \bar{X}^{\prime}, \bar{z}\right)$ $\mathrm{IF}_{\delta^{\prime}}^{\diamond}$ formula $\psi^{\prime \prime}$, where $\delta^{\prime}$ is the order-type over $\bar{y}^{\prime} \oplus \bar{X}^{\prime}$ which is constantly 1 over $\bar{y}^{\prime}$ and restricts to $\delta$ over $\bar{X}^{\prime}$, such that $\psi^{\prime}\left(\bar{x}^{\prime}, \bar{z}\right)=\varphi^{\prime \prime}\left(\bar{\psi} / \bar{y}^{\prime}, \bar{X}^{\prime}, \bar{z}\right)$ where the $\bar{\psi}$ are normal $(\bar{y}, \bar{z})-\mathrm{IF}_{\delta^{\prime \prime}}^{\diamond}$ sentences, where $\delta^{\prime \prime}$ is the restriction of $\delta$ to $\bar{y}$.

Example 2.4. The formula $v X$. $[\square(X \wedge \neg \mu Y \cdot[\diamond(\sim X \vee(Y \vee x))])]$ is an $(x, \varnothing)-\mathrm{IF}_{\delta}^{\square}$ formula for $\delta=(\partial)$, and it is not in normal form. The normalization procedure on this subformula involves surfacing the innermost $\vee$ node, by applying associativity of $\vee$ and distributivity of $\diamond$ over $\vee$, so as to obtain $\neg \mu Y$. [ $\diamond Y \vee(\diamond \sim X \vee$

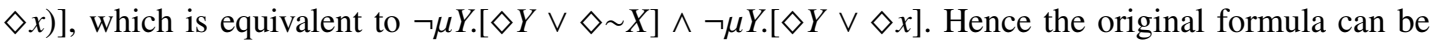

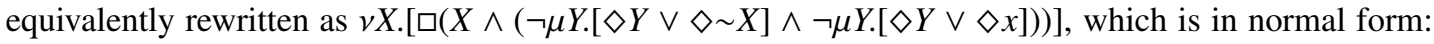
indeed, it is a substitution instance of the formula $v X .\left[\square\left(X \wedge\left(\neg \mu Y .[\diamond Y \vee \diamond \sim X] \wedge y^{\prime}\right)\right)\right]$ which is a $\left(y^{\prime}, \varnothing\right)$ $\mathrm{IF}_{\delta^{\prime}}^{\square}$ with $\delta^{\prime}=(1)$; moreover, $y^{\prime}$ has been substituted for the $\mathrm{IF}^{\square}$ sentence $\neg \mu Y$.[ $\left.\diamond Y \vee \diamond x\right]$.

Proposition 2.5. Every $I F_{\delta}^{*}$ formula, $* \in\{\diamond, \square\}$, is equivalent to an $I F_{\delta}^{*}$ formula in normal form.

\subsection{Adjunction rules for normal inner formulas}

Definition 2.6. For $\bar{x}=\bar{y} \oplus \bar{X}$ of arity $n$, for each order-type $\delta$ over $\bar{x}$, and each $1 \leq i \leq n$, we define maps $\mathrm{LA}_{i}^{\delta}$ and $\mathrm{RA}_{i}^{\delta}$ sending normal $\mathrm{IF}_{\delta}^{\square}$ and $\mathrm{IF}_{\delta}^{\diamond}$ formulas, respectively, into $\mathcal{L}^{+}$, by the following simultaneous recursion: 


$$
\begin{aligned}
& \operatorname{LA}_{i}^{\delta}\left(x_{i}\right)=u \text { for } u \in \operatorname{Var}-(\bar{x} \cup \bar{z}) \\
& \operatorname{LA}_{i}^{\delta}\left(x_{j}\right)=\perp^{\delta_{j}} \text { when } i \neq j \text {; } \\
& \operatorname{LA}_{i}^{\delta}(\square \varphi(\bar{x}, \bar{z}))=\operatorname{LA}_{i}^{\delta}(\varphi)(\diamond u, \bar{z}) ; \\
& \operatorname{LA}_{i}^{\delta}\left(\varphi_{1}(\bar{x}, \bar{z}) \wedge \varphi_{2}(\bar{x}, \bar{z})\right)=\operatorname{LA}_{i}^{\delta}\left(\varphi_{1}\right)(u, \bar{z}) \vee^{\delta_{i}} \operatorname{LA}_{i}^{\delta}\left(\varphi_{2}\right)(u, \bar{z}) ; \\
& \operatorname{LA}_{i}^{\delta}\left(v Y . \varphi\left(\bar{\varphi}(\bar{x}, \bar{z}) / \bar{y}^{\prime}, Y, \bar{z}\right)\right)=\bigvee^{\delta_{i}}\left\{\operatorname{LA}_{i}^{\delta}\left(\varphi_{j}\right)\left(\mu Y .\left[\operatorname{LA}_{j}^{\delta^{\prime}}(\varphi)(Y, \bar{z}) \vee \operatorname{LA}_{j}^{\delta^{\prime}}(\varphi)(u, \bar{z})\right], \bar{z}\right) \mid 1 \leq j \leq \ell\right\} ; \\
& \operatorname{LA}_{i}^{\delta}(\pi(\bar{z}) \rightarrow \varphi(\bar{x}, \bar{z}))=\operatorname{LA}_{i}^{\delta}(\varphi)(u \wedge \pi(\bar{z}), \bar{z}) ; \\
& \operatorname{LA}_{i}^{\delta}(\pi(\bar{z}) \vee \varphi(\bar{x}, \bar{z}))=\operatorname{LA}_{i}^{\delta}(\varphi)(u-\pi(\bar{z}), \bar{z}) ; \\
& \operatorname{LA}_{i}^{\delta}\left(\psi^{c}(\bar{x}, \bar{z}) \rightarrow \pi(\bar{z})\right) \quad=\quad \operatorname{RA}_{i}^{\delta^{\partial}}\left(\psi^{c}\right)(u \rightarrow \pi(\bar{z}), \bar{z}) ; \\
& \operatorname{RA}_{i}^{\delta}\left(x_{i}\right)=u \text { for } u \in \operatorname{Var}-(\bar{x} \cup \bar{z}) \text {; } \\
& \operatorname{RA}_{i}^{\delta}\left(x_{j}\right)=\mathrm{T}^{\delta_{j}} \text { when } i \neq j \text {; } \\
& \operatorname{RA}_{i}^{\delta}(\diamond \psi(\bar{x}, \bar{z}))=\operatorname{RA}_{i}^{\delta}(\psi)(\boldsymbol{\square} u, \bar{z}) ; \\
& \operatorname{RA}_{i}^{\delta}\left(\psi_{1}(\bar{x}, \bar{z}) \vee \psi_{2}(\bar{x}, \bar{z})\right)=\operatorname{RA}_{i}^{\delta}\left(\psi_{1}\right)(u, \bar{z}) \wedge^{\delta_{i}} \operatorname{RA}_{i}^{\delta}\left(\psi_{2}\right)(u, \bar{z}) ; \\
& \operatorname{RA}_{i}^{\delta}\left(\mu Y . \psi\left(\bar{\psi}(\bar{x}, \bar{z}) / \bar{y}^{\prime}, Y, \bar{z}\right)\right)=\bigwedge^{\delta_{i}}\left\{\operatorname{RA}_{i}^{\delta}\left(\psi_{j}\right)\left(v Y \cdot\left[\operatorname{RA}_{j}^{\delta^{\prime}}(\psi)(Y, \bar{z}) \wedge \mathrm{RA}_{j}^{\delta^{\prime}}(\psi)(u, \bar{z})\right], \bar{z}\right) \mid 1 \leq j \leq \ell\right\} ; \\
& \mathrm{RA}_{i}^{\delta}(\psi(\bar{x}, \bar{z})-\pi(\bar{z}))=\operatorname{RA}_{i}^{\delta}(\psi)(\pi(\bar{z}) \vee u, \bar{z}) ; \\
& \operatorname{RA}_{i}^{\delta}(\pi(\bar{z}) \wedge \psi(\bar{x}, \bar{z}))=\operatorname{RA}_{i}^{\delta}(\psi)(\pi(\bar{z}) \rightarrow u, \bar{z}) ; \\
& \operatorname{RA}_{i}^{\delta}\left(\pi(\bar{z})-\varphi^{c}(\bar{x}, \bar{z})\right)=\operatorname{LA}_{i}^{\delta^{d}}\left(\varphi^{c}\right)(\pi(\bar{z})-u, \bar{z}) .
\end{aligned}
$$

By normality, formulas with $v Y$ as main connective are of the form $\left.v Y . \varphi\left(\bar{\varphi}(\bar{y}, \bar{z}) / \bar{y}^{\prime}, \bar{X}, Y, \bar{z}\right)\right)$ where $\varphi\left(\bar{y}^{\prime}, \bar{X}, Y, \bar{z}\right)$ is an $\mathrm{IF}_{\delta^{\prime}}$, formula, with $\delta^{\prime}$ constantly 1 on $\bar{y}^{\prime}$ and $Y$ and restricting to $\delta$ on $\bar{X}$, and the $\bar{\varphi}=\left(\varphi_{1}, \ldots, \varphi_{\ell}\right)$ are $(\bar{y}, \bar{z})-\mathrm{IF}_{\delta}^{\square}$ sentences. Likewise, formulas with $\mu Y$ as main connective are of the form $\left.v Y . \psi\left(\bar{\psi}(\bar{y}, \bar{z}) / \bar{y}^{\prime}, \bar{X}, Y, \bar{z}\right)\right)$ where $\psi\left(\bar{y}^{\prime}, \bar{X}, Y, \bar{z}\right)$ is an $\mathrm{IF}_{\delta^{\prime}}^{\diamond}$ formula, with $\delta^{\prime}$ constantly 1 on $\bar{y}^{\prime}$ and $Y$ and restricting to $\delta$ on $\bar{X}$, and the $\bar{\psi}=\left(\psi_{1}, \ldots, \psi_{\ell}\right)$ are $(\bar{y}, \bar{z})-\mathrm{IF}_{\delta}^{\diamond}$ sentences.

We are now in a position to give versions of the adjunction rules tailored to normal $(\bar{x}, \bar{z})-\operatorname{IF}_{\delta}^{\square}$ and $(\bar{x}, \bar{z})-\mathrm{IF}_{\delta}^{\diamond}$ formulas, for which the adjoints are expressible as $\mathcal{L}^{+}$-term functions:

$$
\frac{\eta \leq \varphi(\bar{x}, \bar{z})}{\&_{i=1}^{n} \operatorname{LA}_{i}^{\delta}(\varphi)[\eta / u] \leq^{\delta_{i}} x_{i}}\left(\mathrm{IF}_{R}\right) \quad \frac{\psi(\bar{x}, \bar{z}) \leq \eta}{\&_{i=1}^{n} x_{i} \leq^{\delta_{i}} \operatorname{RA}_{i}^{\delta}(\psi)[\eta / u]}\left(\mathrm{IF}_{L}\right)
$$

where $\varphi, \eta \in \mathcal{L}^{+}$, the arrays $\bar{x}$ and $\bar{z}$ are disjoint, the arity of $\bar{x}$ is $n, \varphi \in(\bar{x}, \bar{z})-\mathrm{IF}_{\delta}^{\square}$ and $\psi \in(\bar{x}, \bar{z})-\mathrm{IF}_{\delta}^{\diamond}$.

Example 2.7. Consider the inequality $v X .[\square(X \wedge \neg \mu Y$. $[\diamond(\sim X \vee(Y \vee p))])] \leq \diamond \square \neg p$, which is $\epsilon$-recursive for $\epsilon_{p}=\partial$. After first approximation we have:

$$
\forall p \forall \mathbf{i} \forall \mathbf{m}[(\mathbf{i} \leq v X .[\square(X \wedge \neg \mu Y .[\diamond(\sim X \vee(Y \vee p))])] \& \diamond \square \neg p \leq \mathbf{m}) \Rightarrow \mathbf{i} \leq \mathbf{m}] .
$$

No approximation rules are applicable, thus we work toward the application of an appropriate adjunction rule to display the $p$ in the first inequality in the antecedent of the quasi-inequality above. In example 2.4. the normal form of the left hand side of the inequality was computed as, $\varphi=v X$. [ $\square(X \wedge(\neg \mu Y .[\diamond Y \vee$ $\diamond \sim X] \wedge \neg \mu Y$.[ $\diamond Y \vee \diamond p]))]$. Recall that $\varphi$ is a substitution instance of the formula $v X . \varphi^{\prime}=v X .[\square(X \wedge$ $\left(\neg \mu Y\right.$. $\left.\left.\left.[\diamond Y \vee \diamond \sim X] \wedge y^{\prime}\right)\right)\right]$, where $\varphi^{\prime}$ is a $\left(y^{\prime} \oplus X, \varnothing\right)-\mathrm{IF}_{\delta}^{\square}$ formula with $\delta=(1,1)$; moreover, $y^{\prime}$ has been substituted for the $\mathrm{IF}^{\square}$ sentence $\neg \psi=\neg \mu Y$. $[\diamond Y \vee \diamond p]$. Thus, $\operatorname{LA}_{p}^{\epsilon}(\varphi)=\operatorname{LA}_{p}^{\epsilon}(\neg \psi)\left(\mu X\right.$. [ $\operatorname{LA}_{y^{\prime}}^{\delta}\left(\varphi^{\prime}\right)[X / u] \vee$ $\left.\left.\operatorname{LA}_{y^{\prime}}^{\delta}\left(\varphi^{\prime}\right)\right] / u^{\prime}\right)$, where

$$
\begin{aligned}
& \operatorname{LA}_{p}^{\epsilon}(\neg \psi)=\operatorname{RA}_{p}^{\epsilon^{\theta}}(\psi)\left(\neg u^{\prime} / u^{\prime}\right) \\
& =\mathrm{RA}_{p}^{\epsilon^{\theta}}(\mu Y \cdot[\diamond Y \vee \diamond p])\left(\neg u^{\prime} / u^{\prime}\right) \\
& =\left(v Y .\left[\operatorname{RA}_{p}^{\epsilon^{\theta} \oplus 1}(\diamond Y \vee \diamond p)\left[Y / u^{\prime}\right] \wedge \mathrm{RA}_{p}^{\epsilon^{\theta} \oplus 1}(\diamond Y \vee \diamond p)\right]\right)\left(\neg u^{\prime} / u^{\prime}\right) \\
& =\left(v Y \cdot\left[\left(\top \wedge \backsim u^{\prime}\right)\left[Y / u^{\prime}\right] \wedge\left(\top \wedge \backsim u^{\prime}\right)\right]\right)\left(\neg u^{\prime} / u^{\prime}\right) \\
& =\left(v Y \cdot\left[\mathbf{\square} Y \wedge \boldsymbol{\square} u^{\prime}\right]\right)\left(\neg u^{\prime} / u^{\prime}\right) \\
& =v Y \cdot\left[\mathbf{\square} Y \wedge \boldsymbol{\square} \neg u^{\prime}\right] \text {, }
\end{aligned}
$$




$$
\begin{aligned}
\operatorname{LA}_{y^{\prime}}^{\delta}\left(\varphi^{\prime}\right) & =\operatorname{LA}_{y^{\prime}}^{\delta}\left(\square\left(X \wedge\left(\neg \mu Y .[\diamond Y \vee \diamond \sim X] \wedge y^{\prime}\right)\right)\right) \\
& =\operatorname{LA}_{y^{\prime}}^{\delta}\left(X \wedge\left(\neg \mu Y .[\diamond Y \vee \diamond \sim X] \wedge y^{\prime}\right)\right)(\diamond u / u) \\
& =\left(\mathrm{LA}_{y^{\prime}}^{\delta}(X) \vee \mathrm{LA}_{y^{\prime}}^{\delta}\left(\neg \mu Y .[\diamond Y \vee \diamond \sim X] \wedge y^{\prime}\right)\right)(\diamond u / u) \\
& =\left(\perp \vee\left(\operatorname{LA}_{y^{\prime}}^{\delta}(\neg \mu Y .[\diamond Y \vee \diamond \sim X]) \vee \operatorname{LA}_{y^{\prime}}^{\delta}\left(y^{\prime}\right)\right)\right)(\diamond u / u) \\
& =\left(\operatorname{RA}_{y^{\prime}}^{\delta}(\mu Y .[\diamond Y \vee \diamond \sim X])(\neg u / u) \vee u\right)(\diamond u / u) \\
& =(\perp \vee u)(\diamond u / u) \\
& =\diamond u .
\end{aligned}
$$

Now, $\operatorname{LA}_{p}^{\epsilon}(\varphi)=v Y .[\square Y \wedge \square \neg(\mu X .[\diamond X \vee \diamond u])]$. Thus, applying $\left(\mathrm{IF}_{R}^{\sigma}\right)$ to the normalized inequality transforms (2.7) into

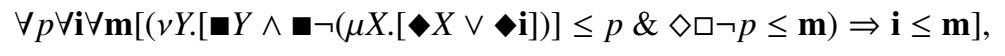

which is in Ackermann shape. Now applying Ackermann's lemma yields the quasi-inequality

$$
\forall \mathbf{i} \forall \mathbf{m}[\diamond \square \neg \nu Y .[\mathbf{\square} Y \wedge \boldsymbol{\mathbf { a }} \neg(\mu X .[\diamond X \vee \diamond \mathbf{i}])] \leq \mathbf{m} \Rightarrow \mathbf{i} \leq \mathbf{m}],
$$

from which all propositional variables have been eliminated.

The second part of the abstract [1] presents a calculus of correspondence for the mu-calculus and the defines the class of recursive formulas.

\section{References}

[1] Willem Conradie, Yves Fomatati, Alessandra Palmigiano, and Sumit Sourabh. Algorithmic correspondence for intuitionistic modal mu-calculus, part 2. Abstract submitted to TACL2013.

[2] Willem Conradie, Silvio Ghilardi, and Alessandra Palmigiano. Unified correspondence. In Logical/Informational Dynamics, in honor of Johan van Benthem, A. Baltag and S. Smets, eds., vol. forthcoming of Trends in Logic: Outstanding Contributions. Springer, 2012.

[3] Willem Conradie and Alessandra Palmigiano. Algorithmic correspondence and canonicity for distributive modal logic. Annals of Pure and Applied Logic, 163(3):338 - 376, 2012.

[4] Willem Conradie, Alessandra Palmigiano, and Sumit Sourabh. Algebraic modal correspondence: Sahlqvist and beyond. Submitted.

[5] Dexter Kozen. Results on the propositional mu-calculus. Theor. Comput. Sci., 27:333-354, 1983.

[6] Johan van Benthem, Nick Bezhanishvili, and Ian M. Hodkinson. Sahlqvist correspondence for modal mucalculus. Studia Logica, 100(1-2):31-60, 2012. 